\title{
Video Article \\ Analyzing the Permeability of the Blood-Brain Barrier by Microbial Traversal through Microvascular Endothelial Cells
}

\author{
Veronika Weber ${ }^{1}$, Kaya Bork ${ }^{1}$, Rüdiger Horstkorte ${ }^{1}$, Heidi Olzscha ${ }^{1}$ \\ ${ }^{1}$ Institute of Physiological Chemistry, Martin Luther University Halle-Wittenberg \\ Correspondence to: Heidi Olzscha at heidi.olzscha@medizin.uni-halle.de
}

URL: https://www.jove.com/video/60692

DOI: doi:10.3791/60692

Keywords: Immunology and Infection, Issue 156, bacterial traversal, blood-brain barrier, cellular adherence, compound screening, endothelial cells, inflammation, meningitis, cellular permeability, postoperative delirium, tight junctions, transfected human brain microvascular endothelial cells

Date Published: 2/14/2020

Citation: Weber, V., Bork, K., Horstkorte, R., Olzscha, H. Analyzing the Permeability of the Blood-Brain Barrier by Microbial Traversal through Microvascular Endothelial Cells. J. Vis. Exp. (156), e60692, doi:10.3791/60692 (2020).

\section{Abstract}

The human blood-brain barrier (BBB) is characterized by a very low permeability for biomolecules in order to protect and regulate the metabolism of the brain. The BBB is mainly formed out of endothelial cells embedded in collagen IV and fibronectin-rich basement membranes. Several pathologies result from dysfunction of the BBB followed by microbial traversal, causing diseases such as meningitis. In order to test the effect of multiple parameters, including different drugs and anesthetics, on the permeability of the BBB we established a novel human cell culture model mimicking the BBB with human brain microvascular endothelial cells. The endothelial cells are grown on collagen IV and fibronectin-coated filter units until confluence and can then be treated with different compounds of interest. In order to demonstrate a microbial traversal, the upper chamber with the apical surface of the endothelial cells is inoculated with bacteria. After an incubation period, samples of the lower chamber are plated on agar plates and the obtained colonies are counted, whereby the number of colonies correlate with the permeability of the BBB. Endogenous cellular factors can be analyzed in this experimental set-up in order to elucidate basic cellular mechanisms of the endothelial cells contributing to the BBB. In addition, this platform allows performing a screen for compounds that might affect the permeability of the endothelial cells. Finally, bacterial traversal can be studied and linked to different pathologies, such as meningitis. It might be possible to extend the model and analyze the pathways of the bacteria through the BBB. In this article, we provide a detailed protocol of the described method to investigate the permeability of the BBB.

\section{Video Link}

The video component of this article can be found at https://www.jove.com/video/60692/

\section{Introduction}

The human BBB is a unique boundary of brain tissue, separating the brain from the blood. It strictly regulates the passage of larger and hydrophilic molecules, blocks paracellular diffusion, and maintains brain homeostasis. It also protects the brain from plasma fluctuations, toxins, microbes, and guides inflammatory cells as part of the central nervous system (CNS) immunity. Since its discovery a century ago ${ }^{1}$, many studies have been carried out to understand the structure and function of the BBB. The complex interactions of cells, proteins, and signals from brain and blood demand still further investigation and models.

The human BBB is composed of three cell types: brain microvascular endothelial cells (BMECs), pericytes, and astrocytes ${ }^{2,3}$. The BMECs differ from the majority of the endothelial cells in the body in that they possess a high number of tight junctions and adherens junctions ${ }^{4}$, low pinocytotic activity ${ }^{2,5}$, and a continuous basement membrane ${ }^{6,7}$ to block paracellular diffusion. Small lipophilic molecules can diffuse and pass the BBB following their concentration gradient; larger and hydrophilic molecules enter or leave the brain only through polarized expressed selective transport systems ${ }^{8}$. This regulation results in a high transendothelial electrical resistance (TEER) of $1,500-2,000 \Omega \cdot \mathrm{cm}^{2}$ that is inversely correlated to permeability ${ }^{9,10}$. Although BMECs build a tight barrier, they can react to local and peripheral signals ${ }^{11,12}$. There is a close interaction between BMECs and astrocytes ${ }^{13}$; the astrocyte end-feet build a layer around the vessels and induce the formation of tight junctions ${ }^{13,14}$. They are involved in BBB maturation with different factors, including transforming growth factor- $\beta$ (TGF- $\beta)^{15,16}$. In addition, pericytes play a key role in the regulation of angiogenesis ${ }^{17}$ and preventing apoptosis of the endothelium in cellular differentiation ${ }^{18}$ (Figure 1). They are embedded in the basement membrane and provide structural stability of the vessel wall ${ }^{19}$. 


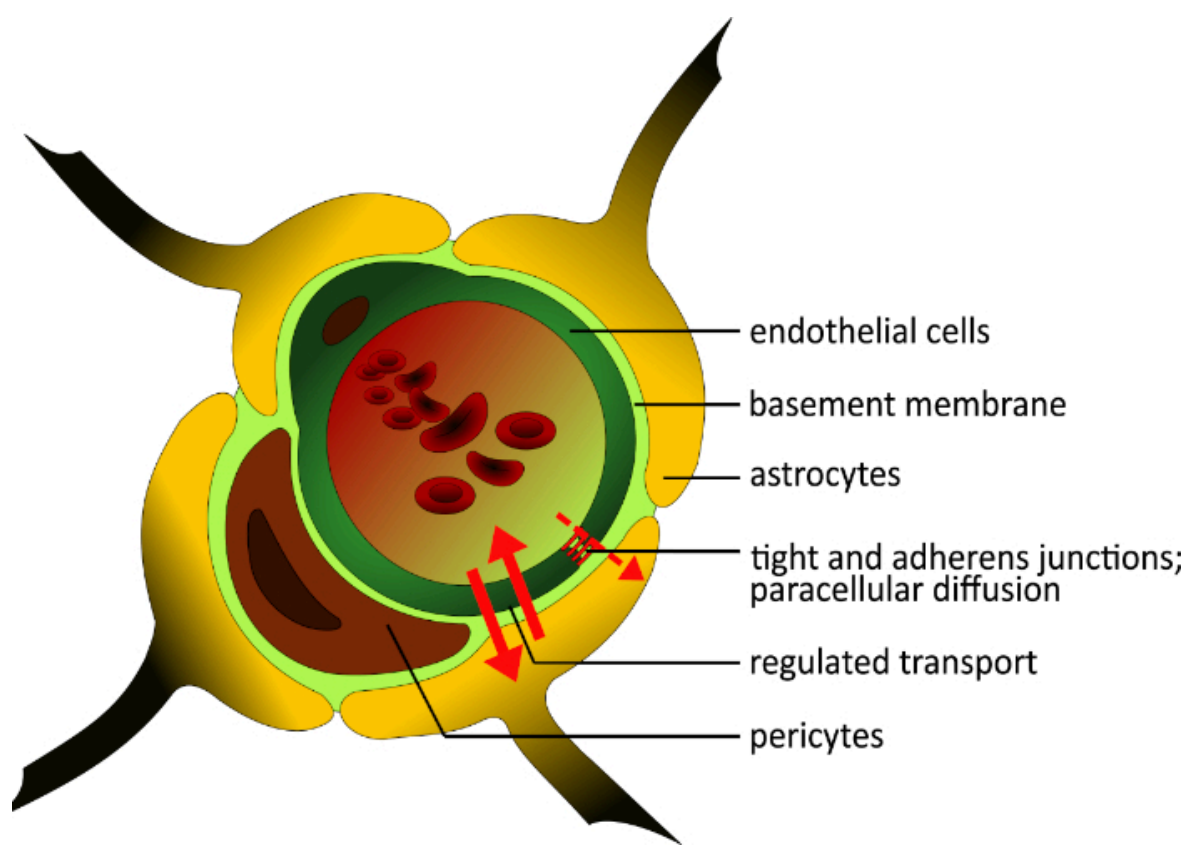

Figure 1: Schematic structure of the blood-brain barrier. The unique structure of the human BBB is composed of three different cell types. The microvessel lumen is surrounded by endothelial cells, which are enriched in tight junctions, and are not fenestrated. They are embedded in the basement membrane, like the pericytes. These cells are important for structural stability of the vessel wall and play a role in the development of the BBB next to the astrocytes. Their end-feet build a close layer around the vessel and support the building of tight junctions. All components of the BBB are important for physiological functionality. Please click here to view a larger version of this figure.

Many different pathologies are related to the collapse of the BBB (e.g., septic encephalopathy). The affected patients have increased protein levels in the cerebrospinal fluid ${ }^{20}$, and the brain parenchyma in affected rodents shows an increased uptake of marked colloidal iron oxide and amino acids ${ }^{21,22}$. These results point towards an increased permeability of the BBB that occurs alongside an increased pinocytosis in BMEC ${ }^{21}$ and endothelial activation ${ }^{23}$. Another associated pathology related to an altered BBB is meningitis, a medical emergency and a complex inflammation accompanied with cerebral edema that can lead to neuronal cell death. The primary entry site of circulating bacteria is supposed to be the microvessels ${ }^{24}$; however, the BBB prevents the entry of bacteria. The permeability of the BBB is not always linked to an increase in experimental hematogenous meningitis ${ }^{25}$ and the mechanisms can be multifactorial. Coincidence of sepsis with postoperative delirium (POD) ${ }^{26}$ and the association with preoperative infections ${ }^{27,28}$ indicates the need for a BBB model that enables the direct exposure to bacteria to get a better understanding into bacterial pathogenesis.

There are many gaps in understanding and quantifying the microbial traversal through the BBB. Therefore, we developed a model that allows a convenient testing of different factors and conditions with a direct correlation between bacterial traversal and influences on the permeability of the BBB. Previous work focused on the paracellular permeability and included TEER measurement and tracer flux. In addition, macromolecule transport was analyzed by conjugated molecules or antibodies, whereby different models using only endothelial cells or combinations with astrocytes and pericytes were developed. Due to the difficulty in obtaining human tissue on a regular basis, many animal-based models are used. Brain endothelial cells of bovine and porcine origin form tight monolayers with a high TEER that form well-shaped apical-basal polarity and are suited for investigations of small molecule transport through the BBB. The proteins differ in sequence from their human homologues ${ }^{29,30}$, making investigation of therapeutic antibodies difficult. For this reason, murine or human culture models may be preferable. Mouse or rats as sample sources have the advantage of being obtained from well characterized species but yield few cells for study purposes. This can be circumvented by the use of immortalized mouse brain endothelioma (END) cell lines bEND.3, bEND.5 or cEND ${ }^{31,32,33}$.

Primary cultured cells from human tissue are difficult to obtain and to handle on a regular basis. Therefore, most human cellular models used in research investigating the human BBB are immortalized endothelial cell lines. A published cell line is human cerebral microvascular endothelial cell line hCMEC/D3, which is well suited for studying drug uptake and is easy to handle. The cells build a monolayer and express the characteristic tight junction proteins of the BBB $^{34}$, whereas the expression level of claudin- 5 is reported to be lower than in intact microvessels ${ }^{35}$ and many specific transporters have been detected at transcript level ${ }^{36}$ as well as in proteomic studies ${ }^{34}$. A relatively low TEER in the range of $30-50 \Omega \cdot \mathrm{cm}^{2}$ is still a challenge ${ }^{37}$. Another source for brain endothelial cells are human pluripotent stem cells (hPSCs) ${ }^{38}$ and human cord blood-derived stem cells of circulating endothelial progenitor and hematopoietic lineages ${ }^{39,40}$. Both protocols of differentiation result in tight cell monolayers and high TEER values (e.g., $1,450 \Omega \cdot \mathrm{cm}^{2}$ in co-cultures) ${ }^{38}$. These stem cell models require extreme care for cultivation, yet offer the opportunity to study the influence of regulating hormones ${ }^{41}$ or diseases with genetic backgrounds ${ }^{42}$ on BBB development.

In this study, we established an immortalized transfected human brain microvascular endothelial cell line, THBMEC ${ }^{43}$, to mimic the BBB and to study bacterial traversal. Cells are seeded on a filter and grown to $100 \%$ confluency in this cell culture model. Bacteria are inoculated in the upper part of the cell culture chamber. We use Escherichia coli $\left(E\right.$. coli) in our sample study because of the high incidence of $E$. coli meningitis ${ }^{44}$. It has been shown that the lowest permeability of cell monolayer occurs between day 13 and day 15 after seeding ${ }^{45}$. Therefore, treatment of the THBMEC monolayer is performed after this time and bacteria are inoculated afterwards in the medium on the apical surface of the monolayer. After an incubation time, bacteria that were able to cross the barrier are quantified via plating medium with the bacteria on agar plates and counting the colonies. An increased number of colonies correlates with higher bacterial traversal through the BBB. The TEER is about $70 \Omega \cdot \mathrm{cm}^{2}$ ${ }^{46}$. However, it is not necessary to measure the TEER in the described method. Although it is a well-established value for the permeability of the 
BBB, it seems to have no impact on the traversal of bacteria through the BBB. Untreated cells serve as a control of tightness in our model. It has been shown in previous work that the cells are able to react to proinflammatory cytokines and express typical tight junction proteins ${ }^{47}$. This allows for compound screening and validation of a larger set of transporter substrates and receptors.

\section{Protocol}

\section{Preparation of Buffer and Reagents}

1. Prepare 10x phosphate buffer saline (10x PBS) by adding $80 \mathrm{~g}$ of sodium chloride $(\mathrm{NaCl}), 2 \mathrm{~g}$ of potassium chloride (KCl), $14.4 \mathrm{~g}$ of disodium-hydrogen-phosphate dihydrate $\left(\mathrm{Na}_{2} \mathrm{HPO}_{4} \cdot 2 \mathrm{H}_{2} \mathrm{O}\right)$ and $2 \mathrm{~g}$ of potassium-dihydrogen phosphate $\left(\mathrm{KH}_{2} \mathrm{PO}_{4}\right)$ in a $1 \mathrm{~L}$ glass flask in $1 \mathrm{~L}$ of double distilled $\mathrm{H}_{2} \mathrm{O}$. Autoclave the 10x PBS solution and dilute $100 \mathrm{~mL}$ of this solution in $900 \mathrm{~mL}$ of double distilled water to get $1 \times$ PBS.

1. Use the autoclave to sterilize solutions. Put the glass flask into the basket, close the lid and sterilize it for $15 \mathrm{~min}$ at $121^{\circ} \mathrm{C}$ and 98.9 $\mathrm{kPa}$.

NOTE: This protocol is always used for autoclaving solutions in further steps.

2. Prepare a $10 \mu \mathrm{g} / \mathrm{mL}$ collagen IV and $10 \mu \mathrm{g} / \mathrm{mL}$ fibronectin solution by diluting $0.5 \mathrm{mg} / \mathrm{mL}$ fibronectin solution and the $0.3 \mathrm{mg} / \mathrm{mL}$ collagen IV solution each with $1 \times$ PBS to $100 \mathrm{mg} / \mu \mathrm{L}$ aliquots in $1.5 \mathrm{~mL}$ micro tubes. Thereafter, mix $100 \mu \mathrm{L}$ of both aliquots with $1,800 \mu \mathrm{L}$ of $1 \times \mathrm{PBS}$ in 2 $\mathrm{mL}$ micro tubes and store them at $-20^{\circ} \mathrm{C}$.

3. Prepare DMEM/F-12 medium by adding $4 \%$ fetal bovine serum and $2 \mathrm{mM} \mathrm{L-glutamine} \mathrm{and} 100 \mathrm{mg} / \mathrm{L}$ penicillin/streptomycin to the medium and store it at $4{ }^{\circ} \mathrm{C}$.

4. Prepare $1 \mathrm{x}$ trypsin-EDTA solution by diluting $5 \mathrm{~mL}$ of the $10 \mathrm{x}$ concentrated trypsin-EDTA solution with $45 \mathrm{~mL}$ of $1 \times$ PBS in a $50 \mathrm{~mL}$ tube and store it at $4{ }^{\circ} \mathrm{C}$.

5. Prepare $500 \mathrm{~mL}$ of LB medium by weighing $10 \mathrm{~g}$ of LB broth base in a $500 \mathrm{~mL}$ glass flask. Add $500 \mathrm{~mL}$ of sterilized water and autoclave it.

6. Prepare LB agar by weighing $10 \mathrm{~g}$ of LB broth base and $7.5 \mathrm{~g}$ of agar-agar in a $500 \mathrm{~mL}$ glass flask. Add $500 \mathrm{~mL}$ of sterilized water before autoclaving and do not close the lid of the flask. Autoclave and leave the solution to cool down until it is warm to the touch.

7. Prepare antibiotic-free medium by adding $4 \%$ fetal bovine serum and $2 \mathrm{mM} \mathrm{L-glutamine,} \mathrm{but} \mathrm{no} \mathrm{penicillin/streptomycin} \mathrm{to} \mathrm{the} \mathrm{DMEM/F-12}$ medium and store it at $4{ }^{\circ} \mathrm{C}$ as in step 1.3 .

\section{Growth of the Blood-brain Barrier Mimicking Cells}

1. To assemble the 12 well plate, put the cell culture inserts into the wells (Figure 2A).

1. Unpack the plate and each insert in a biological safety cabinet and perform further steps there. Use sterilized forceps to grab the insert at its broad base to move it.

2. Coat the porous membrane of each insert with $90 \mu \mathrm{L}$ of $10 \mu \mathrm{g} / \mathrm{mL}$ collagen IV and $10 \mu \mathrm{g} / \mathrm{mL}$ fibronectin mixture. Incubate the $12 \mathrm{well}$ plate for $24 \mathrm{~h}$ at $37^{\circ} \mathrm{C}$ in a cell culture incubator (Figure 2B).

3. Wash the inserts twice by pipetting $1 \mathrm{~mL}$ of $1 \times$ PBS into each insert and aspirate the solution with a vacuum pump for cell cultures.

4. Equilibrate the membranes by pipetting $0.5 \mathrm{~mL}$ of prewarmed DMEM/F-12 medium in the upper and $1.5 \mathrm{~mL}$ in the lower chamber. Incubate the plate for $30 \mathrm{~min}$ at $37^{\circ} \mathrm{C}$ in a cell culture incubator with $5 \% \mathrm{CO}_{2}$ atmosphere (Figure 2C).

5. Seed $2 \times 10^{5}$ human microvascular endothelial cells into each upper chamber and incubate the 12 well plate at $37^{\circ} \mathrm{C}$ in a cell culture incubator (Figure 2D).

1. Aspirate the medium from the cell culture flask using a vacuum pump for cell cultures and wash the monolayer by pipetting $10 \mathrm{~mL}$ of $1 \mathrm{x}$ PBS and aspirating the solution afterwards with a vacuum pump.

2. Cover the cells completely with $1 x$ concentrated trypsin-EDTA by pipetting $5 \mathrm{~mL}$ of the solution into the cell culture flask. Incubate the flask for 3-5 min at $37^{\circ} \mathrm{C}$ in a cell culture incubator.

NOTE: If the cells are not dissociated, firmly tap the flask against the palm of the hand to dislodge the cells.

3. Take $5 \mathrm{~mL}$ with a pipette as an aliquot from the cell suspension in a $15 \mathrm{~mL}$ tube and add $5 \mathrm{~mL}$ of the FCS-containing medium to stop the enzymatic reaction.

4. Centrifuge the suspension for $3 \mathrm{~min}$ at $210 \times \mathrm{g}$, remove the supernatant with a vacuum pump, and resuspend the pellet in $5 \mathrm{~mL}$ of medium with a pipette.

5. Use the cell counter by mixing $10 \mu \mathrm{L}$ of the cell suspension with $10 \mu \mathrm{L}$ of $0.4 \%$ trypan blue stain in a $1.5 \mathrm{~mL}$ micro tube. Add $10 \mu \mathrm{L}$ of the mixture into a counting chamber slide, put it into the cell counter, and start counting.

1. Focus the counter on the cells so that their edge is dark blue and the middle white. Thereafter, start the appropriate program for cell counting.

6. To calculate the volume for each insert, divide the obtained $2 \times 10^{5}$ cells per insert with the calculated concentration of the cell suspension.

\section{Cultivation of the Blood-brain Barrier Model}

1. Incubate the 12 well plate for 14 days at $37^{\circ} \mathrm{C}$.

2. Change $0.5 \mathrm{~mL}$ of medium for the upper chamber and $1.5 \mathrm{~mL}$ of medium for the lower one every $2-3$ days. Warm the medium before putting it in the cell flask. Aspirate with the vacuum pump (Figure 2E).

NOTE: Work carefully to avoid touching the membrane.

3. Check the status of the cells by imaging them with a microscope and determine the confluency. Ensure that the confluency is $100 \%$ after 14 days. 


\section{Preparation of Bacteria}

1. A day before measurement, put a colony of the $E$. coli strain GM2163 into LB medium. Incubate the culture tube for $24 \mathrm{~h}$ at $37^{\circ} \mathrm{C}$ with 180 rpm in an incubation shaker (Figure 2F).

1. Cultivate the E. coli strain on an LB agar plate at $4{ }^{\circ} \mathrm{C}$. Take one colony with a sterilized pick and put the pick in the prepared culture tube with $3 \mathrm{~mL}$ of LB medium.

2. Prepare an LB agar plate for each insert with warm LB agar solution and fill the Petri dishes to half their total volume. Let them become solid and store them at $4{ }^{\circ} \mathrm{C}$.

\section{Treatment of Cells}

1. On day 14 after seeding, treat cells with compounds or measure the transendothelial electrical resistance (TEER), if planned (Figure 2G). NOTE: Always have some untreated cells as a control.

1. To treat cells with the compound of interest, dilute the compound to the final concentration in DMEM/F-12 medium. Add $0.5 \mathrm{~mL}$ of this mixture into the upper chamber and $1.5 \mathrm{~mL}$ into the lower chamber. Incubate the plate in a cell culture incubator for the desired time.

2. Afterwards, exchange the complete medium with antibiotic-free medium by aspirating with the vacuum pump and pipetting (Figure $\mathbf{2 H}$ ).

\section{Measurement of Permeability}

1. To get constant concentrations of bacteria, measure the optical density with a photometer at a wavelength of $600 \mathrm{~nm}$. Dilute the overnight solution of bacteria with $\mathrm{LB}$ medium in a $50 \mathrm{~mL}$ falcon tube to an $\mathrm{OD}_{600}$ of $0.5 \pm 0.05$. Work on ice.

1. Fill $1 \mathrm{~mL}$ of LB medium into a cuvette. Start the photometer and put the cuvette in, marked side forward. Press the bottom "Blank" for measuring the blank value afterwards.

2. Measure the density of the bacterial solution by filling it into a cuvette, putting it in, and pressing the bottom sample. Repeat the measurement during the diluting until obtaining the final concentration.

2. Work in a biological safety cabinet where bacteria can be handled with the prepared 12 well plate and bacterial solution at $O D_{600}=0.5 . A_{d d}$ $450 \mu \mathrm{L}$ of bacterial solution only into each upper chamber containing $0.5 \mathrm{~mL}$ of medium (Figure 2I).

3. Incubate the 12 well plate for $6 \mathrm{~h}$ at $37^{\circ} \mathrm{C}$ in an incubator. NOTE: The protocol dictates to pause here.

4. Sample $50 \mu \mathrm{L}$ of the medium with a pipette from each lower chamber by removing the insert with forceps (Figure $2 \mathrm{~J}$ ). Take care not to spill the medium from the upper chambers to the lower ones.

5. Plate each sample on a separate agar-plate (Figure $\mathbf{2 K}$ ). Drop the sample on the plate and streak out the solution with a cell spreader.

6. Incubate the agar plates for $24 \mathrm{~h}$ at $37^{\circ} \mathrm{C}$ in an incubator.

7. Count the colonies in each plate (Figure $\mathbf{2 L}$ ).

\section{Analyzing Data}

1. Write the data in a table and calculate the average and standard deviation of the observed colonies of treated and untreated cells.

2. Display the average as the absolute number of colonies.

1. To normalize the results, calculate the relative number of colonies by dividing all results with the control value. 
A

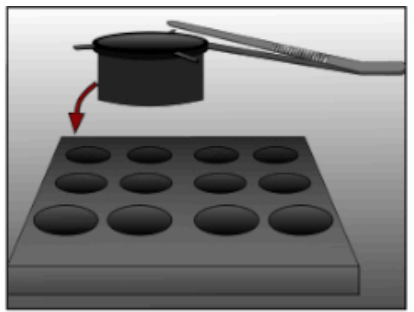

B

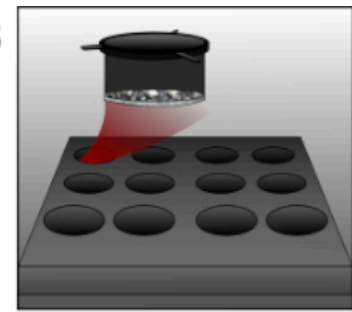

E

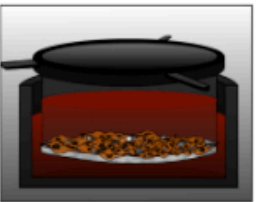

F
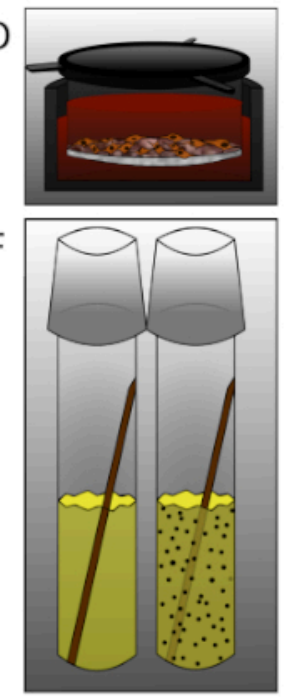

G
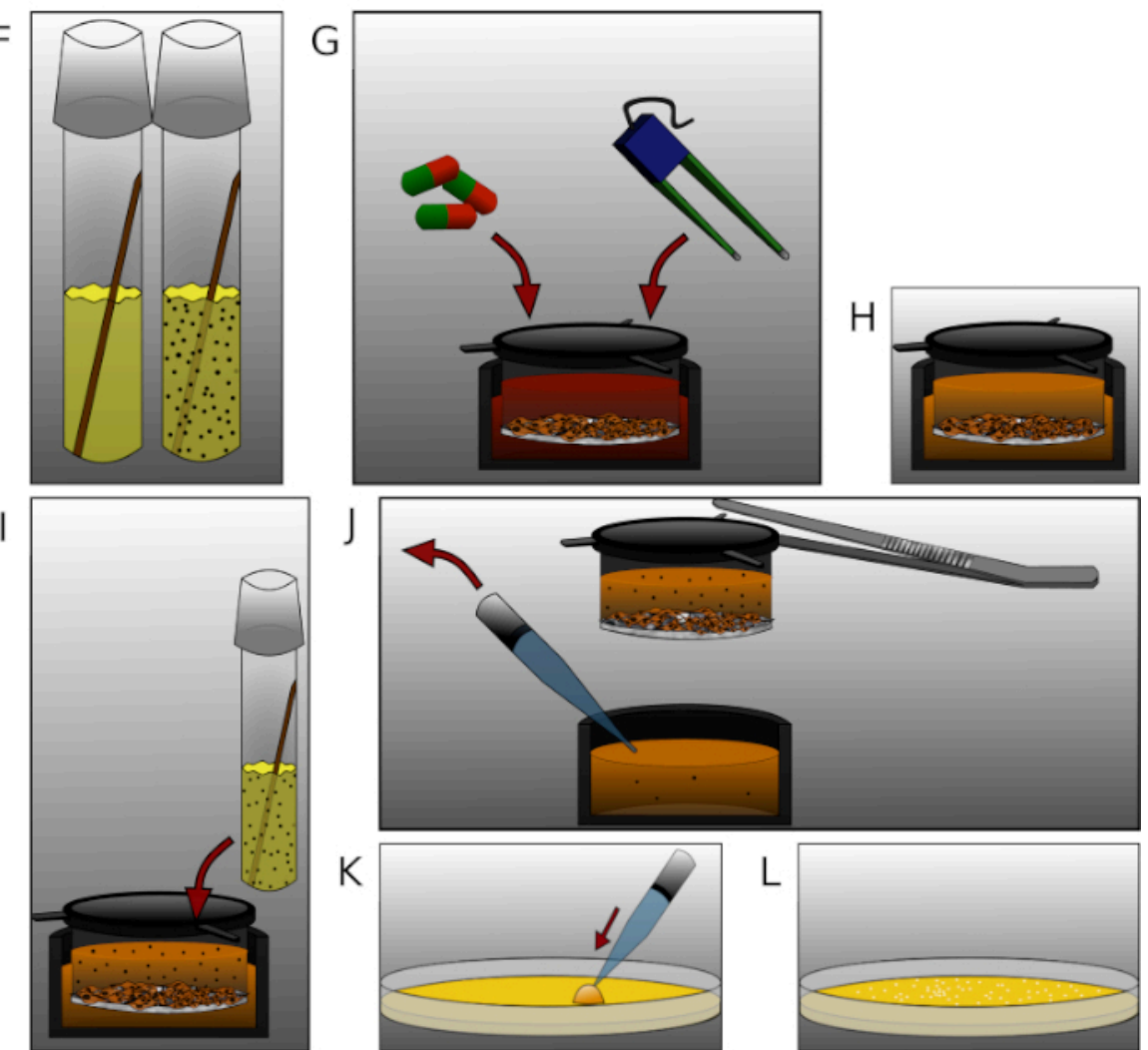

K

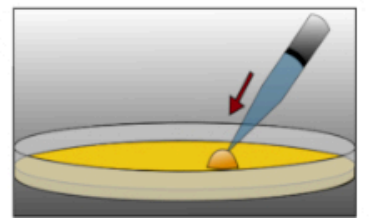

L

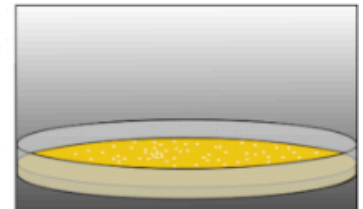

Figure 2: Detailed presentation of the individual steps in the protocol. (A) Put the inserts with sterilized forceps into the 12 well plate. (B) Coat each insert with $90 \mu \mathrm{L}$ of fibronectin and collagen IV mixture and incubate for $24 \mathrm{~h}$. (C) Equilibrate the membranes with prewarmed medium for $30 \mathrm{~min}$. (D) Seed $2 \times 10^{5}$ human brain microvascular endothelial cells per insert. (E) Cultivate the plate for the appropriate amount of time. (F) One day before measuring, put an E. coli colony into a LB medium culture tube and incubate for $24 \mathrm{~h}$. (G) Treat cells or measure TEER. (H) Exchange complete medium with antibiotic-free medium. (I) Add $450 \mu \mathrm{L}$ of bacterial solution $\left(\mathrm{OD}_{600}=0.5\right)$ into each upper chamber and incubate for $6 \mathrm{~h}$. (J) Sample $50 \mu \mathrm{L}$ of medium from each lower chamber removing insert with forceps. (K) Plate the sample on agar plates and incubate for $24 \mathrm{~h}$. (L) Count the colonies and analyze the data. Please click here to view a larger version of this figure. 


\section{Representative Results}

Following the protocol, the cells were seeded, and the BBB model was built. At day 14 after seeding, the cells were treated with glyoxal as a reactive aldehyde. The aim of the experiment was to investigate the correlation between age and diabetes in $\mathrm{POD}^{27}$ and the high incidence of meningitis in elderly patients ${ }^{48}$. The increased levels of advanced glycation end products (AGEs) in both age and diabetes ${ }^{49}$ demand further examination of the effect of glycation in the pathogenesis of microbial traversal through the BBB. Glycation is a non-enzymatic reaction of free amino groups in proteins with carbonyl groups of reducing carbohydrates or other carbonyl compounds. Glucose is well known as donor of carbonyl groups; however, there are more reactive ones known. After building an unstable Schiff's base, they rearrange to more stable and reactive dicarbonyl compounds like glyoxal. AGEs, the final products, can cause crosslinks between proteins ${ }^{50}$. They can damage cellular structures and alter cellular function by interaction with the receptor of AGEs (RAGE) ${ }^{51}$.

Cells were treated with a 0.05 and $0.15 \mathrm{mM}$ glyoxal (GO) solution for $1 \mathrm{~h}$, and untreated cells served as a control. Glycation was detected via immunoblotting and detection with an anti-AGE antibody (Figure 3). The obtained bacterial colonies were counted and represented as the absolute number of colonies (Figure 4A) or the relative number of colonies normalized to the control (Figure 4B). The medium taken from wells with the untreated cells formed very few colonies. This result demonstrated that the untreated cells were able to build a barrier and could serve as a control. Samples treated with glyoxal displayed an increased number of colonies, leading to the conclusion that there is an effect of glyoxal on the THBMECs and the cellular barrier density, because the number of colonies demonstrated a significant difference between untreated and treated cells. The increased bacterial crossing of the barrier after the treatment with glyoxal could explain why diabetes is correlated to diseases with a BBB breakdown.

THBMEC

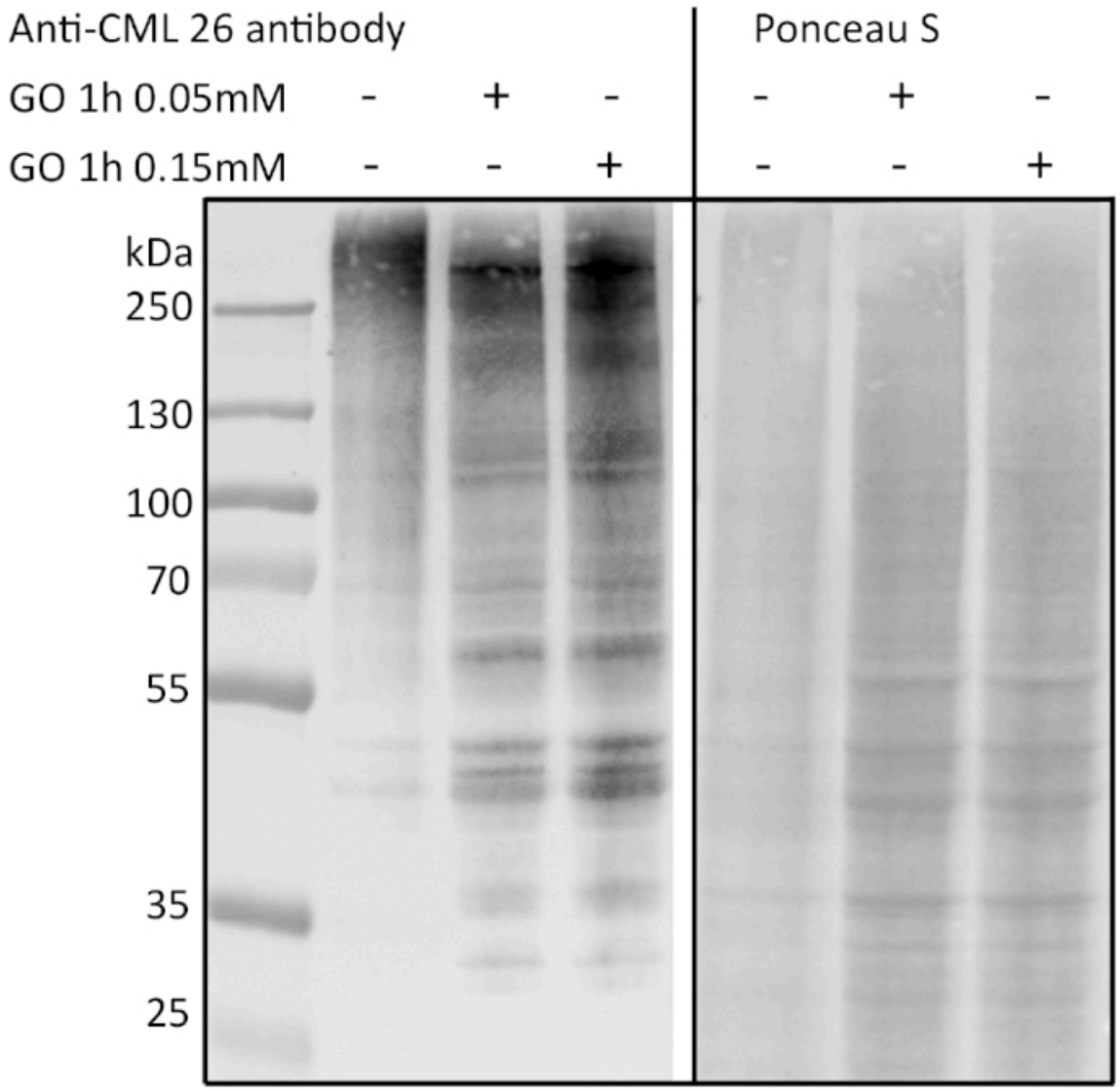

Figure 3: Detection of protein glycation via immunoblotting. THBMECs were treated with GO at different concentrations for $1 \mathrm{~h}$. Total protein was isolated and separated using SDS-PAGE. Glycation of the proteins was detected via immunoblotting using anti-AGE-antibody (CML-26). Please click here to view a larger version of this figure. 
In a different setting, protein glycation of THBMECs was induced by glucose. Sterilized glucose was added to the DMEM/F-12 medium to increase glucose concentration from normal glucose medium (NG) with $17.5 \mathrm{mM}$ to high glucose medium (HG) with $42.5 \mathrm{mM}$. THBMECs were cultivated in two different cell culture flasks: one in normal glucose (NG) medium and the other in high glucose (HG) medium. These two different media were also used for growth of the BBB on filters in 12 well plates. Cells cultivated in NG medium served as a control. The obtained colonies are represented as the absolute number of colonies (Figure 4C) or the relative number of colonies normalized to the control (Figure 4D). The results indicate no significant effect on the traversal of bacteria through the human BBB, leading to the conclusion that the effect of NG vs. HG was not severe enough to affect the integrity of the BBB. The different scenarios were designed to prove the model and the integrity of the cells mimicking the BBB.
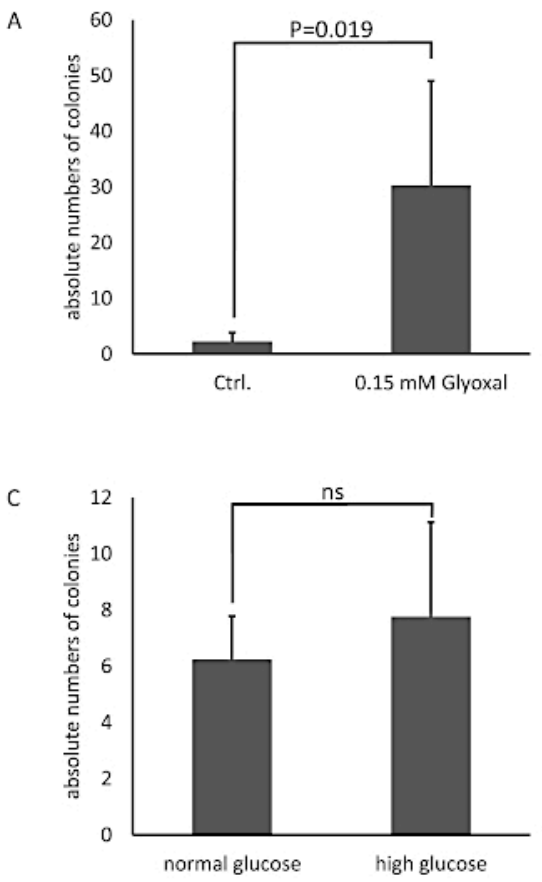

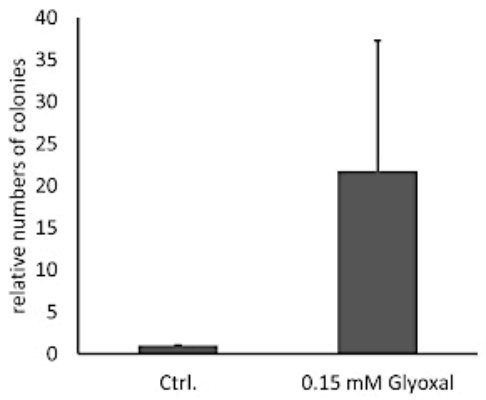

D

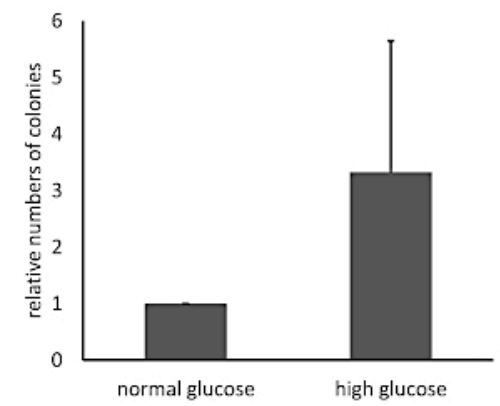

Figure 4: Absolute and relative numbers of counted bacterial colonies in the BBB model with THBMECs. THBMECs were treated with $0.15 \mathrm{mM} \mathrm{GO}$ for $1 \mathrm{~h}$, untreated cells served as a control. A total of $450 \mu \mathrm{L}$ of $E$. coli suspension $\left(\mathrm{OD}_{600}=0.5\right)$ was added to each upper chamber. Medium from the lower chambers was plated on agar plates after $6 \mathrm{~h}$. (A) The graph shows the average mean $+/-$ SEM of counted colonies. (B) The graph shows the counted colonies normalized to the untreated cells as control $+/-$ SEM $(n=4)$. In $(C)$ and $(D)$, THBMECs were cultivated in $\mathrm{NG}$ and $\mathrm{HG}$ medium. A total of $450 \mu \mathrm{L}$ of $E$. coli suspension $\left(\mathrm{OD}_{600}=0.5\right)$ was added to each upper chamber. Medium from the lower chamber was plated on agar plates after $6 \mathrm{~h}$. (C) The graph shows the average mean +/- SEM of counted colonies. (D) The graph shows the counted colonies normalized to the untreated cells as control + - SEM $(n=3)$. Please click here to view a larger version of this figure.

\section{Discussion}

Limited insight into the pathogenesis of microbial traversal limits further development of therapies for POD or meningitis. The mortality and morbidity of these diseases demand better patient treatment, require research of the underlying mechanisms, and need a robust platform for compound screening. The multifactorial events can be studied with human BMECs. Several successful reported isolation procedures of BMECs from a number of species have shown a loss of the cells' characteristics molecular signature ${ }^{52,53}$. The described THBMECs in this procedure were transfected in very early passages, where they exhibited specific brain endothelial cell characteristics and preserved them ${ }^{43}$. This is important, because not all steps in the affected pathways have been discovered so far, and this model seems to mimic conventional BMECs. Our presented model shows direct influences on BMECs and the microbial traversal through the BBB.

The handling of THBMEC cells is straightforward, and the required technical equipment exists in most life science laboratories. Our model allows for an immediate start of investigative procedures after the THBMECs have built a tight monolayer. The field of applications can be extensive because of the possible combinations between new tests and conventional assays such as TEER measurement or labeling with tracers ${ }^{54}$. It is also possible to add astrocytes or pericytes to make a co- or triple-culture model. The influence of drugs on the microbial traversal could also be tested in our model by treating the THBMECs with compounds before inoculating the upper chamber with bacteria. In fact, it is possible to purchase inserts with filters for 96 well plates allowing the automation of the procedure. This can facilitate the implementation of high-throughput drug screening systems to accelerate the discovery of drugs against the mentioned diseases and to reduce side effects on the BBB during drug development.

A critical step in the presented method is the incubation time after adding the bacteria to the upper chamber. It is important to use hours as timelines in the protocol, because the generation time of $E$. coli is only $20 \mathrm{~min}^{55}$. Otherwise, use of different time points could lead to misleading results. There is also a possible risk of contamination between upper and lower chamber during the bacterial exposure if the plates are not handled with care. Any alterations to the 12 well plate at this point could contaminate the medium in the lower chamber. 
E. coli is one well-known, very common cause of bacterial meningitis. Further investigations should test different bacteria that are also associated with meningitis, such as Neisseria meningitidis ${ }^{56}$ or Streptococcus pneumoniae ${ }^{57}$. These seem to use different mechanisms to cross the BBB and need to be better understood for the treatment of patients. In elderly patients, the incidence for POD increases ${ }^{26}$ as well as the number of occurring comorbidities. It is known that there are interactions between different diseases, especially systemic ones like diabetes. In our model, it is possible to simulate those conditions or treat the cells before adding the bacteria.

The model is limited by the direct contact of THBMECs and bacteria, and further research is necessary to investigate potential mechanisms of contact to detect the involved pathways and proteins. However, it is possible to remove inserts and harvest the cells for further analysis. The TEER of the model is lower in comparison to stem cell models ${ }^{38,39,40}$. We confirmed this by using a bacterial concentration that did not cross the BBB in untreated cells after $6 \mathrm{~h}$.

In summary, this method represents a robust platform to analyze the traversal of bacteria through the BBB with the potential to expand it for highthroughput drug screenings.

\section{Disclosures}

The authors have nothing to disclose.

\section{Acknowledgments}

The authors acknowledge Dr. Maryam Hussain for previous work on this method, the group of PD Dr. Kerstin Danker (Charité-

Universitätsmedizin, Berlin) for providing the THBMECs and Juliane Weber for critically reading the manuscript. This study was supported by the RTK 2155 (ProMoAge).

\section{References}

1. Goldmann, E. E. Vitalfärbung am Zentralnervensystem: Beitrag z. Physio-Pathologie d. Plexus chorioideus ud Hirnhäute. (1913).

2. Reese, T. S., Karnovsky, M. J. Fine structural localization of a blood-brain barrier to exogenous peroxidase. Journal of Cell Biology. 34 (1), 207-217 (1967).

3. Risau, W., Dingler, A., Albrecht, U., Dehouck, M. P., Cecchelli, R. Blood-brain barrier pericytes are the main source of gammaglutamyltranspeptidase activity in brain capillaries. Journal of Neurochemistry. 58 (2), 667-672 (1992).

4. Brightman, M. W., Reese, T. S. Junctions between intimately apposed cell membranes in the vertebrate brain. Journal of Cell Biology. 40 (3), 648-677 (1969).

5. Coomber, B. L., Stewart, P. A. Morphometric analysis of CNS microvascular endothelium. Microvascular Research. 30 (1), $99-115$ (1985).

6. Tilling, T., Korte, D., Hoheisel, D., Galla, H. J. Basement membrane proteins influence brain capillary endothelial barrier function in vitro. Journal of Neurochemistry. 71 (3), 1151-1157 (1998).

7. Morris, A. W. et al. Vascular basement membranes as pathways for the passage of fluid into and out of the brain. Acta Neuropathologica. 131 (5), 725-736 (2016).

8. Betz, A. L., Goldstein, G. W. Polarity of the blood-brain barrier: neutral amino acid transport into isolated brain capillaries. Science. 202 (4364), 225-227 (1978)

9. Butt, A. M., Jones H. C., Abbott N. J. Electrical resistance across the blood-brain barrier in anaesthetized rats: a developmental study. Journal of Physiology. 429, 47-62 (1990).

10. Calabria, A. R., Weidenfeller, C., Jones, A. R., de Vries, H. E., Shusta, E. V. Puromycin-purified rat brain microvascular endothelial cell cultures exhibit improved barrier properties in response to glucocorticoid induction. Journal of Neurochemistry. 97 (4), $922-933$ (2006).

11. O'Carroll, S. J. et al. Pro-inflammatory TNFalpha and IL-1beta differentially regulate the inflammatory phenotype of brain microvascular endothelial cells. Journal of Neuroinflammation. 12 (131) (2015).

12. Simi, A., Tsakiri, N., Wang, P., Rothwell, N. J. Interleukin-1 and inflammatory neurodegeneration. Biochemical Society Transactions. 35 (Pt 5 ), 1122-1126 (2007).

13. Janzer, R. C., Raff, M. C. Astrocytes induce blood-brain barrier properties in endothelial cells. Nature. 325 (6101), 253-257 (1987).

14. Tao-Cheng, J. H., Nagy, Z., Brightman, M. W. Tight junctions of brain endothelium in vitro are enhanced by astroglia. Journal of Neuroscience. 7 (10), 3293-3299 (1987).

15. Utsumi, H. et al. Expression of GFRalpha-1, receptor for GDNF, in rat brain capillary during postnatal development of the BBB. American Journal of Physiology and Cell Physiology. 279 (2) (2000).

16. Tran, N. D., Correale, J., Schreiber, S. S., Fisher, M. Transforming growth factor-beta mediates astrocyte-specific regulation of brain endothelial anticoagulant factors. Stroke. 30 (8), 1671-1678 (1999).

17. Balabanov, R., Washington, R., Wagnerova, J., Dore-Duffy, P. CNS microvascular pericytes express macrophage-like function, cell surface integrin alpha M, and macrophage marker ED-2. Microvascular Research. 52 (2), 127-142 (1996).

18. Ramsauer, M., Krause, D., Dermietzel, R. Angiogenesis of the blood-brain barrier in vitro and the function of cerebral pericytes. Faseb Journal. 16 (10), 1274-1276 (2002).

19. Lindahl, P., Johansson, B. R., Leveen, P., Betsholtz, C. Pericyte loss and microaneurysm formation in PDGF-B-deficient mice. Science. 277 (5323), 242-245 (1997).

20. Young, G. B., Bolton, C. F., Archibald, Y. M., Austin, T. W., Wells, G. A. The electroencephalogram in sepsis-associated encephalopathy. Journal of Clinical Neurophysiology. 9 (1), 145-152 (1992).

21. Carlyle Clawson, C., Francis Hartmann, J., Vernier, R. L. Electron microscopy of the effect of gram-negative endotoxin on the blood-brain barrier. Journal of Comparative Neurology. 127 (2), 183-197 (1966).

22. Jeppsson, B. et al. Blood-brain barrier derangement in sepsis: cause of septic encephalopathy? The American Journal of Surgery. 141 (1) 136-142 (1981). 
23. Tighe, D., Moss, R., Bennett, D. Cell surface adrenergic receptor stimulation modifies the endothelial response to SIRS. Systemic Inflammatory Response Syndrome. New Horizons (Baltimore, Md.). 4 (4), 426-442 (1996).

24. Kim, K. S. et al. The K1 capsule is the critical determinant in the development of Escherichia coli meningitis in the rat. Journal of Clinical Investigation. 90 (3), 897-905 (1992).

25. Kim, K. S., Wass, C. A., Cross, A. S. Blood-brain barrier permeability during the development of experimental bacterial meningitis in the rat. Experimental Neurology. 145 (1), 253-257 (1997).

26. Arshi, A. et al. Predictors and Sequelae of Postoperative Delirium in Geriatric Hip Fracture Patients. Geriatric Orthopaedic Surgery and Rehabililation. 9, 2151459318814823 (2018).

27. Smulter, N., Lingehall, H. C., Gustafson, Y., Olofsson, B., Engstrom, K. G. Delirium after cardiac surgery: incidence and risk factors. Interactive CardioVascular and Thoracic Surgery. 17 (5) (2013).

28. Kratz, T., Heinrich, M., Schlauss, E., Diefenbacher, A. Preventing postoperative delirium. Deutsches Arzteblatt International. 112 (17), 289-296 (2015).

29. Uchida, Y. et al. Quantitative targeted absolute proteomics of human blood-brain barrier transporters and receptors. Journal of Neurochemistry. 117 (2), 333-345 (2011).

30. Warren, M. S. et al. Comparative gene expression profiles of $A B C$ transporters in brain microvessel endothelial cells and brain in five species including human. Pharmacology Research. 59 (6), 404-413 (2009).

31. Omidi, Y. et al. Evaluation of the immortalised mouse brain capillary endothelial cell line, b.End3, as an in vitro blood-brain barrier model for drug uptake and transport studies. Brain Research. 990 (1-2), 95-112 (2003).

32. Steiner, O., Coisne, C., Engelhardt, B., Lyck, R. Comparison of immortalized bEnd5 and primary mouse brain microvascular endothelial cells as in vitro blood-brain barrier models for the study of T cell extravasation. Journal of Cerebral Blood Flow and Metabolism. 31 (1), 315-327 (2011).

33. Burek, M., Salvador, E., Forster, C. Y. Generation of an immortalized murine brain microvascular endothelial cell line as an in vitro blood brain barrier model. Journal of Visualized Experiments. (66), e4022 (2012).

34. Ohtsuki, S. et al. Quantitative targeted absolute proteomic analysis of transporters, receptors and junction proteins for validation of human cerebral microvascular endothelial cell line hCMEC/D3 as a human blood-brain barrier model. Molecular Pharmacolgy. 10 (1), $289-296$ (2013).

35. Urich, E., Lazic, S. E., Molnos, J., Wells, I., Freskgard, P. O. Transcriptional profiling of human brain endothelial cells reveals key properties crucial for predictive in vitro blood-brain barrier models. PLoS One. 7 (5), e38149 (2012).

36. Lopez-Ramirez, M. A. et al. Cytokine-induced changes in the gene expression profile of a human cerebral microvascular endothelial cell-line, hCMEC/D3. Fluid Barrier CNS. 10 (27) (2013).

37. Cucullo, L. et al. Immortalized human brain endothelial cells and flow-based vascular modeling: a marriage of convenience for rational neurovascular studies. Journal of Cerebral Blood Flow Metabolism. 28 (2), 312-328 (2008).

38. Lippmann, E. S. et al. Derivation of blood-brain barrier endothelial cells from human pluripotent stem cells. Nature Biotechnology. 30 (8), 783-791 (2012).

39. Boyer-Di Ponio, J. et al. Instruction of circulating endothelial progenitors in vitro towards specialized blood-brain barrier and arterial phenotypes. PLoS One. 9 (1), e84179 (2014).

40. Cecchelli, R. et al. A stable and reproducible human blood-brain barrier model derived from hematopoietic stem cells. PLoS One. 9 (6), e99733 (2014).

41. Lippmann, E. S., Al-Ahmad, A., Azarin, S. M., Palecek, S. P., Shusta, E. V. A retinoic acid-enhanced, multicellular human blood-brain barrier model derived from stem cell sources. Science Reports. 4, 4160 (2014).

42. Lim, R. G. et al. Huntington's Disease iPSC-Derived Brain Microvascular Endothelial Cells Reveal WNT-Mediated Angiogenic and BloodBrain Barrier Deficits. Cell Reports. 19 (7), 1365-1377 (2017).

43. Stins, M. F., Badger, J., Sik Kim, K. Bacterial invasion and transcytosis in transfected human brain microvascular endothelial cells. Microbiological Pathogens. 30 (1), 19-28 (2001).

44. Gaschignard, J. et al. Neonatal Bacterial Meningitis: 444 Cases in 7 Years. Pediatric Infectious Disease Journal. 30 (3), $212-217$ (2011).

45. Hussain, M. The Effect of Glycation on the Permeability of an in vitro Blood-brain Barrier Model. University Halle-Wittenberg, (2015).

46. Weber, V. The effect of glycation on the permeability of human blood-brain barrier. University Halle-Wittenberg, (2019).

47. Hussain, M. et al. Novel insights in the dysfunction of human blood-brain barrier after glycation. Mechanism of Ageing Development. 155, 48-54 (2016).

48. Choi, C. Bacterial meningitis in aging adults. Clinical Infectious Disease. 33 (8), 1380-1385 (2001).

49. Furth, A. J. Glycated proteins in diabetes. British Journal of Biomedical Science. 54 (3), 192-200 (1997).

50. Sajithlal, G. B., Chithra, P., Chandrakasan, G. Advanced glycation end products induce crosslinking of collagen in vitro. Biochimica and Biophysica Acta. 1407 (3), 215-224 (1998).

51. Ray, R., Juranek, J. K., Rai, V. RAGE axis in neuroinflammation, neurodegeneration and its emerging role in the pathogenesis of amyotrophic lateral sclerosis. Neuroscience and Biobehavior Reviews. 62, 48-55 (2016).

52. DeBault, L. E., Cancilla, P. A. Gamma-Glutamyl transpeptidase in isolated brain endothelial cells: induction by glial cells in vitro. Science. 207 (4431), 653-655 (1980).

53. Diglio, C. A., Grammas, P., Giacomelli, F., Wiener, J. Primary culture of rat cerebral microvascular endothelial cells. Isolation, growth, and characterization. Laboratory Investigation. 46 (6), 554-563 (1982).

54. Buchert, M., Turksen, K., Hollande, F. Methods to examine tight junction physiology in cancer stem cells: TEER, paracellular permeability, and dilution potential measurements. Stem Cell Reviews. 8 (3), 1030-1034 (2012).

55. Gibson, B., Wilson, D. J., Feil, E., Eyre-Walker, A. The distribution of bacterial doubling times in the wild. Proceedings of the Royal Society B: Biological Sciences. 285 (1880), (2018).

56. Pron, B. et al. Interaction of Neisseria maningitidis with the components of the blood-brain barrier correlates with an increased expression of PilC. Journal of Infectious Diseases. 176 (5), 1285-1292 (1997).

57. Iovino, F., Orihuela, C. J., Moorlag, H. E., Molema, G., Bijlsma, J. J. Interactions between blood-borne Streptococcus pneumoniae and the blood-brain barrier preceding meningitis. PLoS One. 8 (7), e68408 (2013). 